\title{
Les Cahiers de Wallis-et-Futuna
}

\section{Raymond MAYER}

\section{(2) OpenEdition}

\section{Journals}

\section{Édition électronique}

URL : http://journals.openedition.org/jso/2092

DOI : $10.4000 /$ jso.2092

ISSN : 1760-7256

\section{Éditeur}

Société des océanistes

\section{Édition imprimée}

Date de publication : 15 décembre 2008

Pagination : 341-343

ISBN : 978-2-85430-012-3

ISSN : 0300-953x

\section{Référence électronique}

Raymond MAYER, «Les Cahiers de Wallis-et-Futuna », Journal de la Société des Océanistes [En ligne], 126-127 | Année 2008, mis en ligne le 01 décembre 2008, consulté le 24 septembre 2020. URL : http:// journals.openedition.org/jso/2092 ; DOI : https://doi.org/10.4000/jso.2092

Ce document a été généré automatiquement le 24 septembre 2020

(c) Tous droits réservés 


\title{
Les Cahiers de Wallis-et-Futuna
}

\author{
Raymond MAYER
}

\section{RÉFÉRENCE}

Les Cahiers de Wallis-et-Futuna, Futuna, revue annuelle éditée par l'Association des Amis de Wallis-et-Futuna, six numéros parus de 2001 à 2006.

1 Une revue dans le Pacifique qui se veut à la fois locale, annuelle et scientifique, et qui en est à sa sixième parution, est à l'échelle des îles Wallis-et-Futuna un événement et mérite quelque peu le détour. On doit à deux enseignants du collège de Sisia (circonscription d'Alo, île Futuna), Oddon Abbal, docteur en histoire de l'université de Montpellier 3, directeur de publication, et Marc Soulé, doctorant en géographie de l'université Paris 1 , de donner vie à une revue modeste (72 pages annuelles) mais régulière qui s'attache à promouvoir l'analyse endogène et l'écriture des ressortissants des îles.

2 Le numéro 1 (année 2001) commence (pp. 6-12) par le commentaire historique d'une notice ancienne consacrée à l'île Futuna (1889) et s'achève (pp.39-70) par une bibliographie de l'histoire de Wallis-et-Futuna postérieure à la Deuxième Guerre mondiale. Ces deux contributions sont de la plume de l'historien Frédéric Angleviel. Les deux autres articles du premier numéro portent sur un récit étiologique de maladie «Moso et ses fils » par Dominique Pechberty (pp. 14-17), ainsi qu'une analyse critique du meurtre du Père Chanel (pp. 18-34) par Oddon Abbal sous le titre suggestif « Faut-il réhabiliter Musumusu?». Figurent aussi les recensions (pp.35-38) de la thèse de doctorat soutenue en 1999 par Adriano Favole à l'université de Turin (Ko le launiu. La palma del potere. I capi e la costituzione della società a Futuna (Polinesia Occidentale), 289 p.) et de l'ouvrage collectif 101 mots pour comprendre Wallis et Futura, dirigé par Atoloto Malau, Atonio Takasi et Frédéric Angleviel, et publié aux éditions île de Lumière à Nouméa.

3 Sur l'ensemble de ce numéro fondateur, on pourra ergoter sur quelques défauts dans la mise en œuvre typographique (par exemple la délicieuse coquille transformant, p. 40, 
l'adjectif «nouveaux» en "no'Uveaux», ou le non-respect de l'espacement conventionnel entre un tiret et le nom qui le suit). On pourra aussi discuter le parti pris de l'auteur de ne pas avoir, suivant ses propres termes «intégré à ce travail la littérature grise, du fait de la quasi-inaccessibilité des rapports de l'ORSTOM-CNRS ou des rapports internes des différents services administratifs », chacun sachant qu'une bonne historiographie a tout à gagner de la consultation d'une documentation sans limitations a priori, a fortiori quand celle-ci relève de sources d'expertise). Mais le premier numéro avait donné le ton sur l'essentiel et l'exigence d'une production de niveau soutenu était inscrite dans les faits.

4 Le numéro 2 de mars 2002 capte l'intérêt du lecteur à cause de la distance critique que les articles instaurent par rapport à la situation locale. C'est ainsi que l'histoire du père Henquel, qui est la bible sociale et foncière de Wallis, est analysée par Petelo Leleivai (pp. 15-28) du point de vue des enjeux politiques des familles régnantes et des relations entre Wallis et Tonga, ce qui a pour effet de relativiser le point de vue quelque peu dogmatique généralement exercé par cet ouvrage de référence dans la fixation de la tradition oralo-écrite de l'île Wallis. Dans la même perspective, un enseignant futunien, Atonio Takasi, traite des représentations historiques liées à "Osokalaga : guerrier et homme politique " de la fin du XVIII ${ }^{\mathrm{e}}$ siècle (pp.6-14). Ce deuxième numéro s'illustre surtout par l'intérêt scientifique inédit porté aux « Lotu mavae: identité religieuse en mutation à Wallis-et-Futuna » par Filihau Asi Talatini (pp. 29-55), l'expression lotu mavae signifiant littéralement "religion qui s'éloigne " (de la religion catholique qui a régné sans partage depuis 1842 sur l'archipel) et se rapportant à l'irruption sur la scène wallisienne et futunienne d'obédiences chrétiennes ne se réclamant pas du catholicisme, nommément l'Église évangélique, les Témoins de Jéhovah, l'Église évangélique de Pentecôte et l'Église de Jésus-Christ des Saints des Derniers Jours. Diplômé de l'InALCO, Filihau Asi Talatini offre une analyse qualitative et chiffrée d'un phénomène qui a débuté dans la diaspora wallisienne et futunienne de NouvelleCalédonie dès les années 1970 et qui révolutionne depuis le milieu des années 1980 le paysage religieux des îles-mères. Le numéro est complété par un état des « risques majeurs à Futuna» par Marc Soulé (pp.56-67) et une recension du catalogue de l'exposition organisée en 2001 par Daniel Frimigacci et Bernard Vienne à Nouméa sous le titre Wallis-Futuna, 3000 ans d'histoire, Nouméa, Association de la jeunesse wallisienne et futunienne, $63 \mathrm{p}$. (recension par Odon Abbal).

5 Le numéro 3 de mars 2003 revient à une écriture plus européenne en publiant les articles "Néotectonique et géomorphologie de Futuna», extrait d'un mémoire de maîtrise des sciences de la Terre de Noël Gourmelen (pp.6-35, illustré de 26 photographies en couleur), "Relations homme/récif corallien sur l'île de Wallis " extrait d'un mémoire de géographie de Sybylle Eschapasse (pp. 36-51), et « Coutume et cultures à Futuna: un lien indissociable » à partir du mémoire de géographie sur le point d'être soutenu par Marc Soulé (pp. 52-68). Un compte rendu du livre bilingue français-anglais publié par le Service des affaires culturelles de Futuna à Suva, sous la direction d'Elise Huffer et Petelo Leleivai Futuna aux deux royaumes (Futuna mo ona puleaga sau) est signé de Daniel Estebe.

6 Avec le numéro 4 de mars 2004 apparaît un changement de maquette de couverture qui arbore désormais une photographie illustrant l'un des thèmes de la revue, en l'occurrence, pour la jaquette du numéro 4, une photographie ancienne en sépia présentant deux garçons wallisiens censés apprendre le chant grégorien. De fait, un 
article sur "Les danses et les chants à 'Uvea" analysées par Dominique Pechberty (pp. 54-66) introduit au domaine musical dans la revue, tandis que Frédéric Angleviel consacre pour sa part deux articles à l'histoire contemporaine des îles : «Wallis-etFutuna durant le premier septennant de F. Mitterrand (1981-1988): nouveaux dynamismes et permanences insulaires» (pp. 6-32) et «Éphémérides (1981-1988)» (pp. 33-53). Le même présente la parution dans la collection Fac-similés océaniens aux éditions L'Harmattan de l'ouvrage Wallis-et-Futuna: Aux temps premiers de la Mission (1841-1862). Extraits des Annales pour la Propagation de la Foi (édition commentée et annotée par Odon Abbal, 2004, 220 p.). Une recension du mémoire de maittrise de géographie soutenu en janvier 2004 à l'université Paris Iv par Marc Soulé sous le titre «La place de la coutume dans l'organisation de l'espace et dans la société sur l'île de Futuna » (en voir l'article présenté plus haut dans le numéro 3) achève le numéro 4.

7 La couverture du numéro 5 de mars 2005 affiche une « inattendue » vue aérienne en couleur de l'archipel Clipperton. C'est que la contribution de Christian Jost, en poste à l' unc de Nouméa, porte exceptionnellement (pp.58-86) sur l'histoire et la géographie de cet atoll qui n'a évidemment rien à voir avec le terrain wallisien et futunien, mais qui offre des perspectives comparatives susceptibles d'applications locales. Par ailleurs, Dominique Pechberty et Frédéric Angleviel reviennent avec des articles respectivement dédiés à la médecine traditionnelle à 'Uvea (pp. 6-22) - aspect également abordé par le kinésithérapeute Augustin Schoch (pp.23-29) - et à l'histoire de l'éducation par l'Église catholique à Wallis-et-Futuna sur la période 1837-1961 (pp. 30-57). Christophe Jacquet signe une brève recension de l'ouvrage collectif paru pour le compte du ministère de l'Outre-mer et du gouvernement de la Nouvelle-Calédonie aux éditions ScerenL'Harmattan L'Outre-mer français dans le Pacifique, Nouvelle-Calédonie, Polynésie française, Wallis-et-Futuna, 2003, $282 \mathrm{p}$.

8 Le numéro 6 de juillet 2006 donne en photographie de couverture des enfants de Kolopelu (Futuna) agitant de petits drapeaux tricolores à bouts de bras à l'occasion du 14 juillet 2005. Au sommaire du numéro figure un article conséquent d'un doctorant des sciences de l'éducation, Soane Vehika, sur « L'enfant wallisien et futunien en milieu familial et scolaire» (pp. 6-43). L'auteur, responsable du service territorial des œuvres scolaires et de la vie de l'étudiant, examine les principales modalités d'interactions et d'apprentissage en double référence au milieu familial insulaire et aux conceptions du milieu scolaire, en souhaitant que d'utiles conclusions puissent améliorer les pratiques en vigueur. Le directeur de la publication, Odon Abbal, propose quant à lui deux études. La première porte sur «Les mythes de Wallis-et-Futuna » (pp. 44-58) et y souligne la pérennité des genres fakamatala et fanaga de même que la constance des thématiques d'un corpus qui continue à servir de révélateur de la psychosociologie insulaire. La seconde est le commentaire d'un fac-similé du premier article consacré à lîle Futuna par le Bulletin de la Société de Géographie (juillet-décembre 1842) à partir des écrits missionnaires tirées des Annales de la Propagation de la Foi diffusées alors, comme le rappelle Odon Abbal, à « 16000 exemplaires » (pp. 59-64), ce qui peut laisser rêveur un lecteur de modeste revue de "société savante "! La livraison du numéro 6 des Cahiers de Wallis-et-Futuna s'achève sur la recension par Odon Abbal du livre de Dominique Pechberty Vivre la coutume à 'Uvea (Wallis) publié en collaboration avec Epifania Toa, professeur de langues régionales, aux éditions L'Harmattan, 2005, 326 p. Enfin Augustin Schoch fait état d'un livre de 192 pages " premier ouvrage rédigé par des Wallisiens comportant six thèmes de la vie locale : chefferie, langue locale, éducation, coutume, 
religion et agriculture. L'ouvrage bilingue français-anglais est le résultat d'une collaboration entre l'université du Pacifique Sud à Suva (Fidji) et le service des affaires culturelles de Wallis ». On ne saurait souhaiter meilleure continuation à cette production locale qui a toute sa raison d'être.

\section{AUTEUR}

\section{RAYMOND MAYER}

université Lyon II 